PHYSICAL REVIEW D 94, 089901(E) (2016)

\title{
Erratum: First-order post-Newtonian analysis of the relativistic tidal effects for satellite gradiometry and the Mashhoon-Theiss anomaly \\ [Phys. Rev. D 93, 044057 (2016)]
}

Peng $\mathrm{Xu}$ and Ho Jung Paik

(Received 27 September 2016; published 6 October 2016)

DOI: 10.1103/PhysRevD.94.089901

We would like to make the following amendments to the paper:

1. In Eqs. (11a), (11b), (11c), and (11d), the indices of the matrix $K^{N}, K^{G E}, K^{G M}$, and $K^{L T}$ must all be lower indices. The correct expressions should be $\left(K^{N}\right)_{\mu \nu}$ in Eq. (11a), $\left(K^{G E}\right)_{\mu \nu}$ in Eq. (11b), $\left(K^{G M}\right)_{\mu \nu}$ in Eq. (11c), and $\left(K^{L T}\right)_{\mu \nu}$ in Eq. (11d).

2. In Eq. (11d), the $(3,3)$ element is 0 , which is missed.

3. In Eq. (22b), the $(2,3)$ and $(3,2)$ elements must be replaced by

$$
-\frac{1}{4} \sin i[\Psi(\cos 2 \Psi+1)+\sin 2 \Psi]
$$

We are grateful to Neil Ashby for pointing out the typos in Eq. (22b). 